\title{
Experimental Characterisation of the Far-Field Noise in Axial Fans Fitted with Shaped Tip End-Plates
}

\author{
S. Bianchi, ${ }^{1}$ A. Corsini, ${ }^{1}$ and A. G. Sheard ${ }^{2}$ \\ ${ }^{1}$ Dipartimento di Ingegneria Meccanica e Aerospaziale, Sapienza University of Rome, 00184 Rome, Italy \\ ${ }^{2}$ Fläkt Woods Ltd, Colchester CO4 5ZD, UK \\ Correspondence should be addressed to A. G. Sheard, geoff.sheard@flaktwoods.com
}

Received 29 December 2011; Accepted 24 January 2012

Academic Editor: S. C. M. Yu

Copyright ( $) 2012$ S. Bianchi et al. This is an open access article distributed under the Creative Commons Attribution License, which permits unrestricted use, distribution, and reproduction in any medium, provided the original work is properly cited.

\begin{abstract}
The authors investigate the far-field noise emissions of a datum fan blade fitted with tip end-plate geometries, originally designed to control the leakage vortex swirl level. The end-plate geometries influence the tip-leakage flow, vortex formation, and swirl level. In doing so, the end-plate geometries influence the sound-power levels. After an evaluation of fan rotors' aerodynamic performance, the study compares the rotors' far-field noise signature characterised in terms of sound-power and pressure-level spectra to enable and assess the end-plate acoustic pay-off. The investigation attempts to establish a cause-and-effect relationship between the tipflow dynamics and the radiated sound fields, exploring the diverse directivity patterns. The authors found a tonal reduction, due to the enhanced blade-tip end-plates and clarified the relevance of the tip features influencing the radial distribution of the noise sources using coherence analysis. The modified multiple-vortex breakdown end-plate design was effective in reducing the broadband noise, giving an improvement in the frequency range of the turbulent noise.
\end{abstract}

\section{Introduction}

Researchers have studied the link between the aerodynamic features of the fan rotor and its acoustic emissions extensively. In particular, Wright [1] and Cumpsty's [2] works have enhanced the understanding of axial-turbomachinery aeroacoustics. Cumpsty [2] concluded that, with the exception of the low-frequency range of high-speed machines, the mechanism that determines broadband noise in subsonic fans is the same as that in supersonic tip-speed fans and compressors. According to Wright [1], this is due to the prominence of rotor noise originating from turbulent boundary layers.

Researchers have identified a variety of mechanisms as causing noise signatures. The dominant sources, they believe, are the rotor blades, which generate noise as a result of turbulent wake shedding from the interaction between the end-wall boundary layer and the rotor tip. In view of the aerodynamic effect that tip-leakage flow exerts on wake and secondary flows, the industry widely recognises this mechanism as one of the most significant sources of noise $[3,4]$.
The advent of stringent environmental regulations with respect to noise production has stimulated academics and practitioners alike to pursue the development of concepts and technologies that are likely to reduce fan noise either by attenuating noise propagation or by controlling the noise at source. In this regard, researchers have not given the deserved attention to the control and reduction of noise that the rotor-tip flow field generates primarily because of a lack of understanding of the complex flow fields involved. Nonetheless, some studies have addressed this question.

Marcinowski [5], who was the first scholar to study tipgenerated noise, demonstrated that increases in broadband noise levels occur with increasing tip-clearance, with the largest changes apparent at frequencies greater than the blade passing frequency. Mugridge and Morfey [6] argued that an optimum tip clearance exists at which broadband noise is at a minimum due to the countervailing effects of the tip-clearance flow and the blade passage vortex. However, Longhouse [7] did not confirm this result as he searched for a practical solution to the tip-clearance noise emitted by cooling fans and concluded that the cause emanated from the unstable blade-tip vortex which impacted the adjacent blade 
pressure side. Longhouse obtained the lowest noise levels with the smallest possible tip clearance. Fukano and Jang [8] reported similar findings, whereas Kameier and Neise's [9] experiments demonstrated that, with the smallest possible tip clearance, noise reduced over a limited frequency range, close to the blade passage frequency. However, random noise actually increased.

During the past decade, several researchers have proposed passive noise-control concepts based on modifications to the blade tip by means of antivortex appendages. Quinlan and Bent [10] have proposed end-plates, and others have proposed various solutions in patents for industrial fans [1114]. The present study focuses on a family of contemporary commercially available fans. Utilising an experimental technique for the spanwise detection of rotor noise sources [15], the study compares the aeroacoustic performance of the datum blade (which is typical of a class of low-noise industrial fans) with two proposed tip configurations: an improved configuration (designated TF in the present study) developed by the Fläkt Woods Group [16] and a new tip appendage with variable thickness (designated $M V B$ in the current study) [17]. To this end, Figure 1 summarises the historical timeline of the blade-tip end-plate design, from the datum configuration to the $M V B$.

The objective of the study is to compare the acoustic merits of the proposed tip configurations, which, according to Corsini et al.'s [18] quantitative study, are capable of controlling tip-leakage flow and vortex formation, thus producing benefits in terms of an overall reduction in sound power levels. The comparative investigation focuses on the assessment of the emitted noise in the far field speculating on the variation of the overall sound power level with the fan's operating condition, that is, the aerodynamic power. Moreover, the authors explore the directivity patterns by plotting angular maps of the narrowband sound pressure level spectra 0 to 90 degrees off the fan axis. The investigation attempts to establish a cause-and-effect relationship between the tip-flow dynamics and the sound field radiation patterns.

The paper organisation is as follows. First, it describes the methodology in detail, including a description of (i) the family of fans under investigation (ii) passive noisecontrol devices (iii) flow conditions (iv) experimental setup (v) aerodynamic performance measurements, and (vi) far field acoustic measurements. The paper next presents the authors' findings, including (i) main aerodynamic and acoustic performance analysis and (ii) rotor noise sources' directivity analysis. The paper concludes with a discussion of the findings and a summary of the major conclusions.

\section{Methodology Outline}

The authors designed the study's methodology to achieve the following specific objectives: (i) to characterise various fanblade configurations' azimuthal distribution of the radiated sound pressure $\left(L_{p}\right)$ and power levels $\left(L_{w}\right)$ and (ii) to investigate the directivity of the $L_{p}$ spectra and speculate the noise origin.

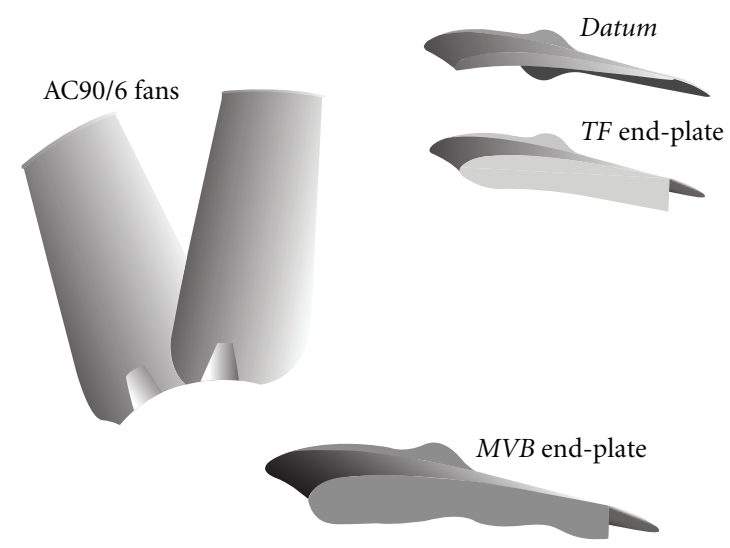

Figure 1: Test fan rotor blades and tip end-plates (not to scale) [17].

TABLE 1: Specifications of AC90/6 fan family of fans.

\begin{tabular}{|c|c|c|}
\hline \multirow[b]{2}{*}{ Blade geometry } & \multicolumn{2}{|c|}{ AC90/6 fans } \\
\hline & Hub & Tip \\
\hline $1 / t$ & 1.32 & 0.31 \\
\hline Pitch angle (deg) & 36 & 28 \\
\hline Camber angle (deg) & 46 & 41 \\
\hline solidity & 1.24 & 0.3 \\
\hline \multicolumn{3}{|l|}{ Fan rotor } \\
\hline Blade number & \multicolumn{2}{|c|}{6} \\
\hline Blade tip pitch angle (deg) & \multicolumn{2}{|c|}{$16 / 28$} \\
\hline Blade tip stagger angle (deg) & \multicolumn{2}{|c|}{$74 / 62$} \\
\hline Hub-to-casing diameter ratio $v$ & \multicolumn{2}{|c|}{0.22} \\
\hline Tip diameter $(\mathrm{mm})$ & \multicolumn{2}{|c|}{900.0} \\
\hline Rotor tip clearance $\chi(\%$ span $)$ & \multicolumn{2}{|c|}{1.0} \\
\hline Rated rotational frequency (rpm) & \multicolumn{2}{|c|}{$935 / 950$} \\
\hline
\end{tabular}

The authors conducted the study on a family of cooling fans, coded AC90/6, featuring good acoustic performance with respect to the state of the art according to in-service experience. The investigated fans were equipped with a sixblade unswept rotor, with modified ARA-D-geometry-type blade profiles originally designed for propeller applications. Table 1 provides specifications of the datum fan AC90/6.

2.1. Fans and Tip End-Plates. The authors studied a datum fan that engineers at Fläkt Woods previously developed, with a view to low noise emission, together with the authors' own improved end-plate configurations (designated $T F$ and $M V B$ ), as Figure 1 illustrates. Although not drawn to scale, Figure 1 provides a qualitative comparison of the thickness distributions of the improved tip concepts against the datum baseline.

The improved blade-tip configurations of the TF class of fans were originally inspired by concepts that researchers developed to control tip vortex and reduce induced drag in aircraft wings or catamaran hulls. Corsini and coworkers $[17,19]$ designed the TF end-plates in accordance with the theory behind vortex control in aircraft wings; that is, they 
determined end-plate dimensions in proportion to the radial dimension of the leakage vortex that they wished to control, estimated in this case as 0.1-0.2 blade spans in tip-limited low-speed rotors of axial compressors [20] and fans [21].

With a view to correcting the TF rotor's negative feature which caused a loss in fan efficiency, Corsini and Sheard [19] had first proposed an alternative blade-tip configuration that included an end-plate of variable thickness. In addition, they have recently developed and tested a second end-plate design concept $[15,22,23]$. The combination of the variable thickness principle concept allowed the realisation of a new tip end-plate, called MVB (multiple-vortex breakdown), as patented by Corsini et al. [17]. In accordance with the background experiences on tip leakage vortex swirl behaviour, this second end-plate design criterion advocates the linkage between the end-plate geometry and the modulation of tip leakage vortex near-axis swirl. The authors interpret this direct linkage flow-to-noise control by deliberately designing the shape of the end-plate at the tip to control the chord wise evolution of the leakage vortex rotation number.

2.2. Experimental Technique. The authors conducted the experiments in the Fläkt Woods semianechoic chamber at Colchester (UK). The chamber is a state-of-the-art anechoic facility used to certify noise emissions from industrial fans according to the BS 848-2.6:2000 standard, which is equivalent to the ISO 10302:1996 standard. The chamber cut-off frequency is $25 \mathrm{~Hz}$.

The authors balanced the rotor to ensure vibration no greater than $4 \mathrm{~mm} / \mathrm{s}$, which is the accepted maximum value for this family of fans. Clearance between the blade tips and the casing was constant at $1 \%$ of blade span. Motor drove the rotor at a constant speed of $940 \mathrm{rpm}$, and the blade tip speed was $44.34 \mathrm{~m} / \mathrm{s}$. Under these conditions, the blade passing frequencies (BPFs) for all the tested configurations were $95 \pm 1.5 \mathrm{~Hz}$. The authors conducted preliminary tests to isolate the motor spectral signature so as to allow for a correction of subsequent noise measurements.

The test-rig was a standardised airway of type-A testing, Figure 2, having the fan downstream from the plenum chamber with a free outlet in an arrangement pertinent to compact cooling fan installations. The rotor centre line was 2 meters from the floor. The bell mouth, on the fan inlet, was aerodynamically optimised to give uniform and unseparated flow into the fan. The authors acoustically treated the downstream and upstream plena to minimise transmission of incidental noise from the air stream and the outside environment. The airflow passed through an airfoil louver in the top of the inlet section of the facility. This louver enabled the authors to make variations in the fan head and flow rate.

The authors set the microphone 2 meters from the rotor trailing edge plane. During the measurements, the authors protected the microphone diaphragm with a nose-cone windshield, upon which they conducted a preliminary test to quantify its self-induced noise, which thus enabled them to include a correction factor in data-processing calculations. The authors recorded signals from the far field microphone

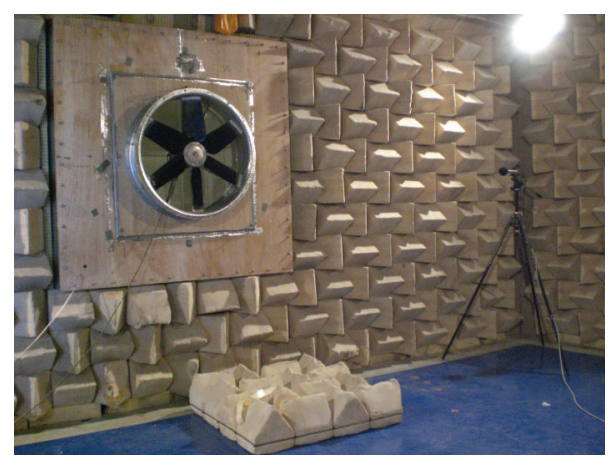

FIGURE 2: Test rig set-up in the semianechoic room.

on separate channels of a 01-dB Symphonie digital signal processor. In all cases, the authors measured signals for 30 seconds. They repeated each measurement three times with an error band $\pm 1 \%$.

The authors measured the far field noise two fan diameters from the outflow sections, as recommended by Leggat and Siddon [24], for measurements in a semianechoic environment. They measured the noise at an angle of 30 degrees from the centre line, according to the directivity characteristics of axial flow fans [24]. Air-speed measurements at the location of the far field microphone revealed only a negligible airflow for all the tested operating conditions. The authors set the far field microphone at the same height as the fan centreline.

\section{Results}

3.1. Investigated Flow Conditions. The authors investigated the fan rotor blades with a large tip-pitch angle of 28 degrees (when measured in the peripheral direction). This constituted the customary setting for these fans. The study accounted for a full performance test, in the way prescribed by the British Standard 848, spanning the operating range of the fan unit, that is, $V=5-9 \mathrm{~m}^{3} / \mathrm{s}$ and $\Delta p_{\text {tot }}=200-310 \mathrm{~Pa}$.

The authors then examined the influence of the different end-plates on the fans' noise emission by examining the noise performance when fitted with each of the three blade-tip configurations (datum, TF, and MVB) under semianechoic conditions. Finally, the experimental test matrix included simultaneous measurements of near-field and far field noise operation point which was customary in operating this family of fans: $\Delta p_{\text {tot }}=280 \mathrm{~Pa}$.

The tests established the aerodynamic performance of the three rotors. Figure 3 illustrates the rotors' operating margins in terms of (a) total pressure rise and (b) the efficiency based on total pressure rise. The authors measured the aerodynamic performance for the fans under standard air condition. It is evident from Figure 3(a) that the $M V B$ outperforms the other two geometries, reaching a higher peak pressure.

As a consequence of its higher-pressure-developing capability, the MVB stall occurred abruptly, whereas the passage from the normal condition to stall was fairly smooth with 


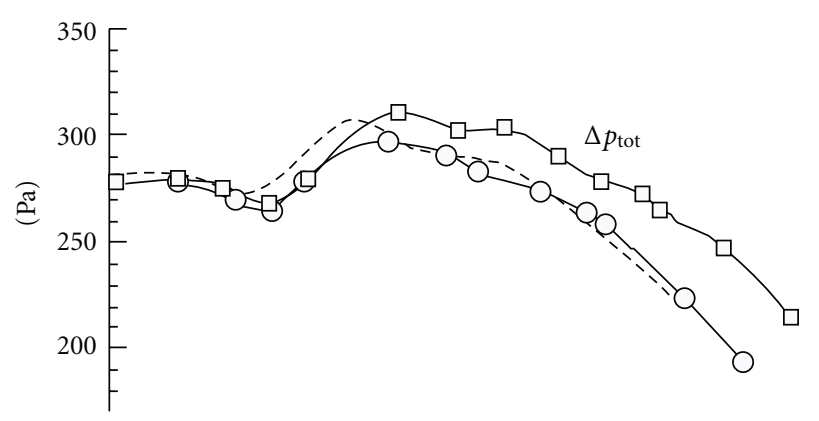

(a)

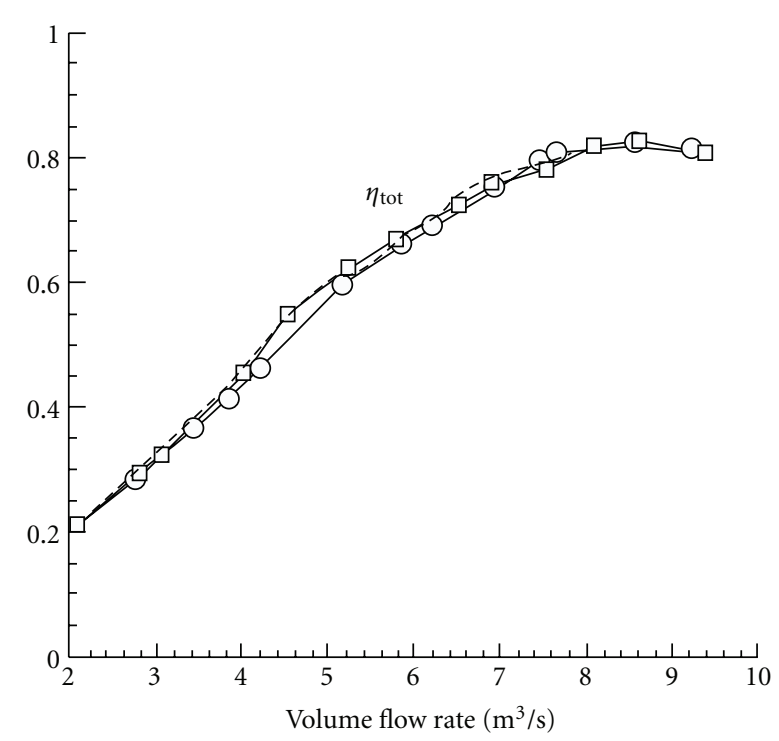

(b)

Figure 3: Comparison of (a) total pressure rise and (b) total efficiency curves.

the datum blade and blade fitted with the TF end-plate. Moreover, the comparison of the total efficiency, Figure 3(b), highlighted that the efficiency of the three rotors was similar.

3.2. Acoustic Performance. To compare the acoustic performance in the far field, first, Figure 4 shows the sound-powerlevel $\left(L_{w}\right)$ spectra for the different blades at $280 \mathrm{~Pa}$, close to the datum fan design operating condition. In particular, Figure 4 shows the A-filtered one-third octave band $L_{w}(A)$ spectra in semilogarithmic scale.

Compared with the datum blade, the TF configuration produced a significant reduction of $L_{w}$ in the broadband signature which $M V B$ further decreased. Notably, the $M V B$ novel tip configuration was effective also in reducing the dominant low-order harmonics. Quantitatively, the MVB configuration outperformed the other configurations in providing a reduction of $5 \mathrm{~dB}$ in the second BPF harmonic. Looking at this occurrence, Bianchi et al. [22] showed that, according to Magliozzi et al. [25] and Cumpsty [2], the second BPF might also be evidence of inflow disturbances from the plenum that were capable of affecting the end-wall

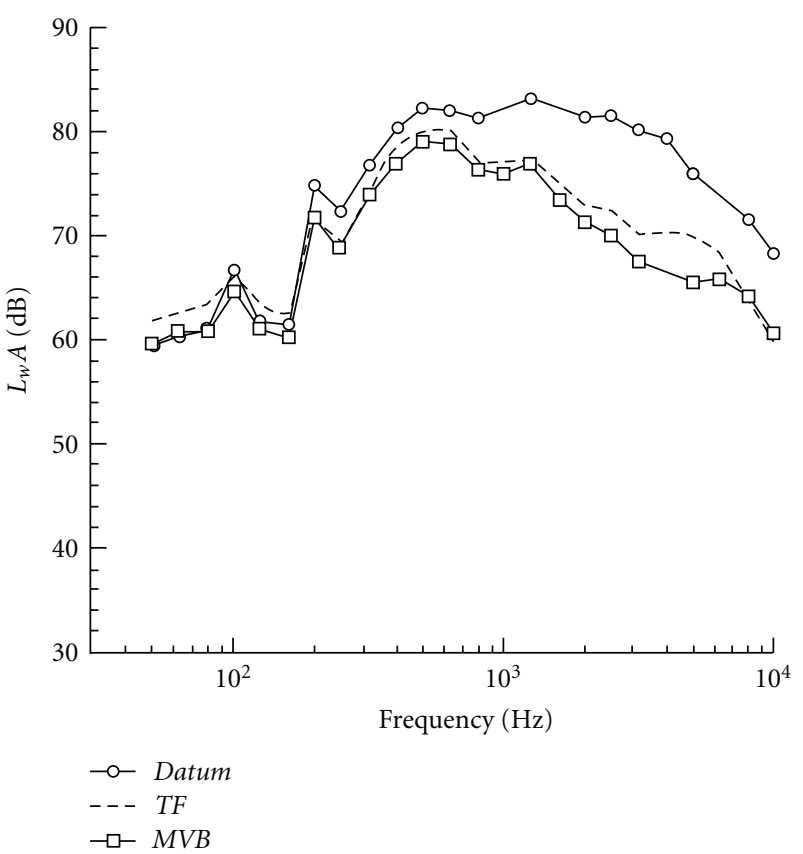

Figure 4: Comparison of the A-filtered one-third octave band sound-power-level auto-spectra.

boundary layer and interaction with the blade's trailing edge turbulent wake.

The authors achieved the larger noise reduction produced by the $M V B$ configurations in the broadband, in a range from $1.5-10 \mathrm{kHz}$, where a significant drop of $L_{w}(A)$ spectrum took place. According to Corsini et al. [18], this drop is likely due to the end-plate's action in disrupting and mixing high-scale turbulence in a large number of lowscale vortices rotating at higher frequencies. The reduction of the rotor aeroacoustic signature, both in terms of tonal noise and broadband noise, demonstrates the effectiveness of the improved tip concepts, with either baseline or variablethickness end-plates. These noise components are related to the main recognised tip noise generation mechanisms in axial decelerating turbomachinery. It is possible to link the convection of tip vortices responsible primarily for the tonal noise, whilst the tip vortex instabilities relate to the creation of broadband self-generated noise [26].

To provide additional insight into the map of acoustic performance when spanning the fan operating margin, Figure 5 shows polar diagrams correlating the A-filtered overall sound power level $\left(O L_{w}(A)\right)$ and the specific noise level $\left(K_{s}\right)$ when varying the airflow power in the fan operating range. Figure 5 shows the pay-off in the $M V B$ noise emission when compared with the datum and the TF rotors.

Notably, the datum fan features a unique behaviour entailing, when increasing the power, a reduction of the specific noise level and an augmented sound power level $O L_{w}(A)$. This evidence suggests that the increased aerodynamic share in the sound generating mechanism, due to the actual fan operations, radiates more efficiently to the far field and enhance the emitted noise in the audible frequency 


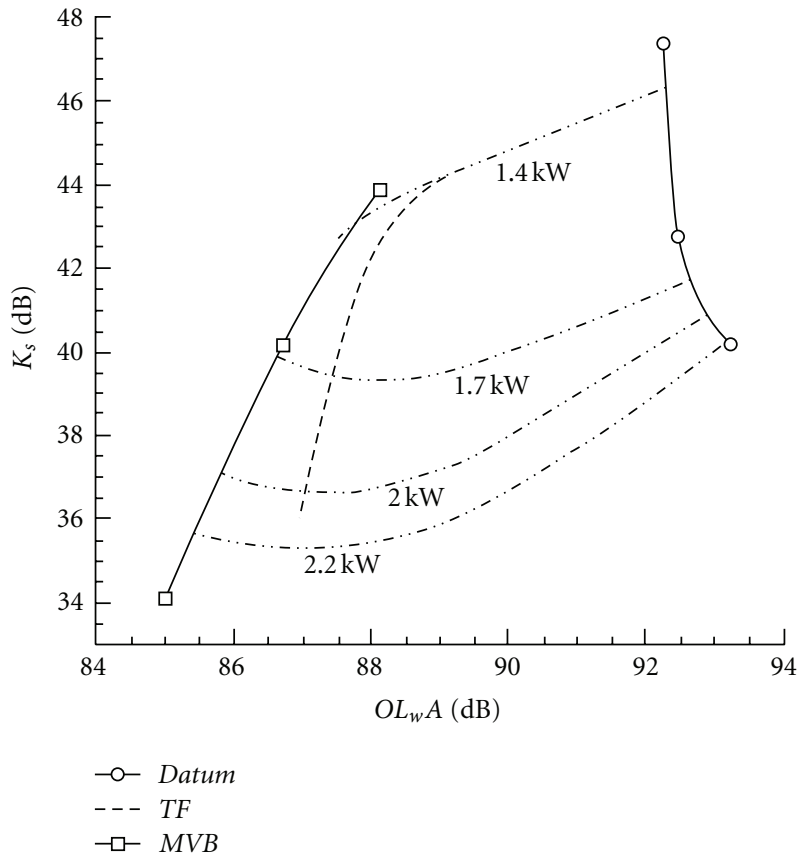

Figure 5: Comparison of A-filtered overall sound power level $\left(O L_{w}(A)\right)$ - specific noise level $\left(K_{s}\right)$ polar plot.

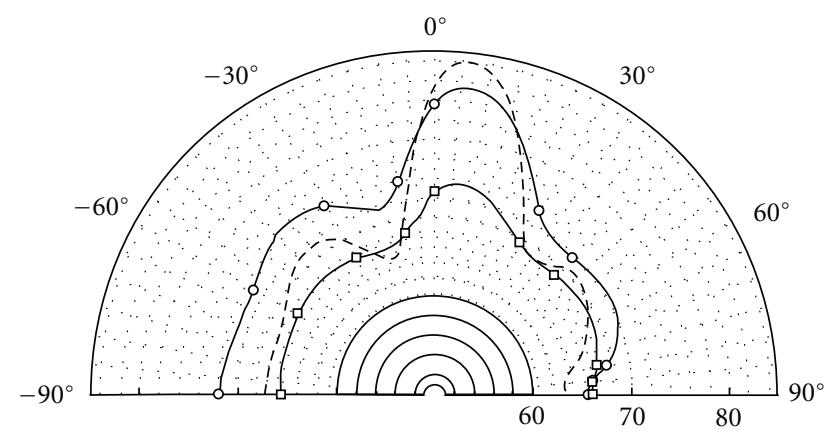

$(\mathrm{dB})$

$$
\begin{aligned}
& \multimap-\text { Datum } \\
& --- \text { TF } \\
& \rightarrow-M V B
\end{aligned}
$$

Figure 6: Directivity of the integrated $L_{p}$ for the different blade geometries.

range. In contrast, both the fan blades fitted with end-plates show a difference in the sound-power-specific power levels. In particular, the $T F$ end-plate shows that the reduction of specific noise level with aerodynamic power correlates to an attenuation of the emitted noise according to a power law $K_{s} \propto O L_{w}(A)^{n}$ with $n=0.5$. However, the MVB speedsup the reduction in sound levels featuring a nearly linear distribution of exponent $n=1$.

Table 2 provides evidence of the noise reduction potential of the proposed original tip concepts by comparing the unweighted and A-weighted $O L_{w}$ at different operating points. This data set refers to hemispherical acoustic measurements that the authors performed according to BS 848 standard. It is evident that both the configurations markedly outperform the datum fan. When comparing the $M V B$ performance to the datum ones, remarkably, the hemispherical noise data record $4 \mathrm{~dB}(\mathrm{~A})$ diminution at the highest flow rate and $8 \mathrm{~dB}(\mathrm{~A})$ diminution in near-peak pressure operation.

3.3. Far Field Directivity Analyses. The authors derived the $L_{p}$ autospectra of the instantaneous pressure signals in the far field under the tested operating condition and then integrated it to calculate the $L_{p}$ azimuthal radiation patterns at seven angular positions on the rotor axis plane. Figure 6 shows the integrated $L_{p}$ value of the datum fan plotted against those of the rotors fitted with the end-plates. It is evident that the peak levels aligned with the fan axis for the datum and TF fans. Remarkably, the MVB fan features a nearly isotropic radiation pattern with a large attenuation (as compared to all the other fan blades) of the axial level. As expected [24], the maxima in the near-axis direction for the datum and TF fans indicated a significant dipolar noise source in the fan outlet. In the near-axis region, $T F$ had a maximum $L_{p}$ level of $82 \mathrm{~dB}$, similar to the datum one $(81 \mathrm{~dB})$, whereas the $M V B$ peak was at $71 \mathrm{~dB}$. According to previous directivity studies in a different test rig set-up [27], the tendency toward the attenuation of in-axis noise correlated with the presence of coherent swirling structures in the exhaust flow. TF noise control design is even more magnified in the MVB end-plates.

When moving away from the fan axis, all the modified impellers' directivity patterns differed from that of the datum fan. While the datum and the TF fans featured a dipolarlike emission, the $M V B$ directivity confirmed its isotropic radiation. In a jet-noise sense, the $M V B$ far field directivity is a consequence of low-frequency noise sources (radiating axially) shifting to the high-frequency range (radiating laterally) which we can consider as the acoustic counterpart of the control implemented on the tip-leakage vortex (i.e., enhanced mixing) and shed vorticity (i.e., reduction of three-dimensional blade separation). This interpretation is routed on the causal relationship between small-scale random turbulent structures and high-frequency sound with nearly isotropic radiation, and large-scale coherent eddies with directional patterns according to their frequency scales.

The analyses showed that the datum's dipolar noise characteristic and original TF rotors changed in the $M V B$ as a result of the superposition of reduced intensity longitudinal dipole and a lateral quadrupole. As Shah et al. [28] previously stated, we can attribute this quadrupole-like noise source to the turbulent mixing of the swirling core flow which scatters the dipolar source related to the presence of a coherent vortical structure.

In order to give hints on the directivity patterns of the far field noise, Figures 7-9 show the $L_{p}$ autospectra mapped in the frequency range $50 \mathrm{~Hz}$ to $5 \mathrm{kHz}$ changing the azimuthal direction from 0 - to 90 -degrees angle to the fan axis. 


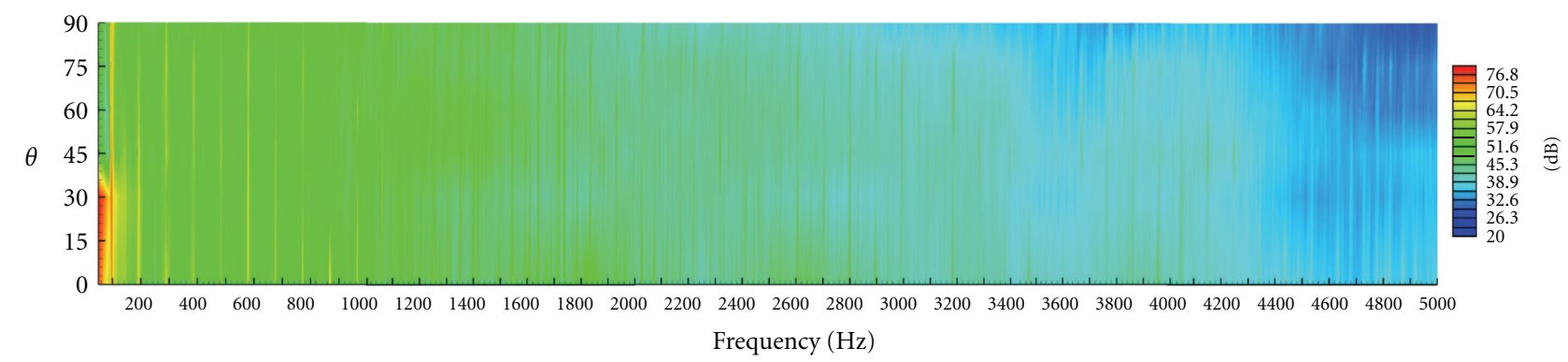

Figure 7: Directivity map of the $L_{p}$ far field autospectra for the datum fan.

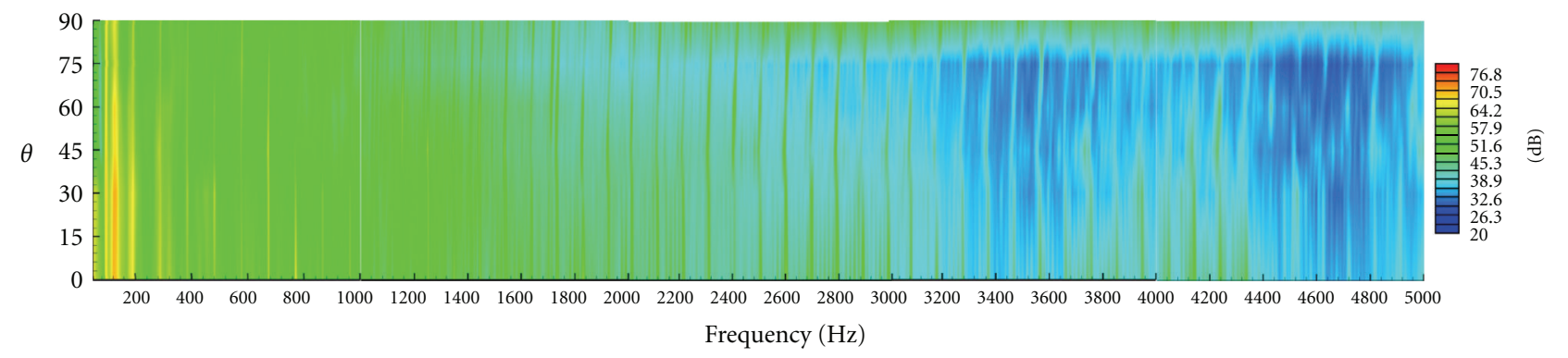

Figure 8: Directivity map of the $L_{p}$ far field autospectra for the TF fan.

Figure 7 shows the $L_{p}$ far field autospectra directivity map for the datum fan. Although the authors confirmed that datum fan noise emission was mainly anisotropic, both in the tonal as well as in the broadband noise components, some features departing from this picture are evident. The authors recorded the major acoustic emissions at low frequency in the vicinity of the fan centreline, in accordance with the particular dipolar-like noise signature found in early studies on low-speed fans [24]. The tonal character of the far field noise dominates the spectral map up to $1 \mathrm{kHz}$. The dominant first BPF harmonic (at $96.8 \mathrm{~Hz}$ ) radiates isotropically as it does at a reduced $L_{p}$, the sixth harmonic. The higher-order tones appeared to radiate coaxially in the angular sector $\theta=$ $0-35$ degrees, but also with a significant side radiation $\theta=$ 50-75 degrees at a reduced sound pressure level. Notably, intertonal BPF sidebands are evident up to 30 degrees off the axial direction. The map shows the evidence of a background noise comprised in a sound pressure level interval 60-50 dB radiating uniformly up to $1.2 \mathrm{kHz}$. At the higher frequency, in contrast, the broadband $L_{p}$ decreases, but features a peculiar radiation pattern with two preferred directions, for example, 60 degrees and close to 0 degrees. Above $2 \mathrm{kHz}$, the sound pressure level rapidly dropped to $30 \mathrm{~dB}$, with peaks located on the fan axis. Due to this broadband noise reduction, higher-order harmonics emerge. First, they were directed all around the azimuthal sector and then with some tones localised from $\theta=45-90$ degrees in the range comprised between $f=3.5-3.7 \mathrm{kHz}$. BPF harmonics were still evident up to $4.2 \mathrm{kHz}$ at $\theta=60$ degrees and then merely broadband noise characterised the datum's noise emission. In this frequency range the intertone level was about $40 \mathrm{~dB}$ and the broadband level was constant at about $30 \mathrm{~dB}$.

When comparing the TF map, Figure 8, to the datum, it is apparent that the radiated noise's spectral characteristics were relatively unchanged in the low-frequency range up to the fourth BPF harmonic. The low frequency tones in the sector of $\theta=0-40$ degrees showed a main reduction in their pressure level, as it happened for the first two BPF harmonics, if compared with the datum. These two harmonics were evident all around the considered sector, but after the third harmonic the emitted noise was mainly broadband. Few tone spots remained from frequencies comprised between the third and the eighth BPF harmonics, radiating mainly toward the sector of $\theta=0-30$ degrees. After $1.4 \mathrm{~Hz}$, some tones emerged due to the gradual reduction of the broadband noise level (as happened for the datum, but here more clearly). These tones remained in evidence for all the considered frequency ranges and radiated in the first quarter of the measured azimuthal sector. After $4.3 \mathrm{kHz}$, these tones merge in the broadband, the level of which varied from $25-30 \mathrm{~dB}$ in the side angles to $38-40 \mathrm{~dB}$ in the on-axis position. The differences from the datum were mainly a minimal favourite pattern of the $L_{p}$ emission in the fan's side zone (at $\theta=90$ degrees) for the tonal structures distributed around the first BPF harmonic and a general decrease on-axis in the high frequency range.

The MVB map (Figure 9), in contrast with the datum and state-of-the-art end-plate maps featured a marked 


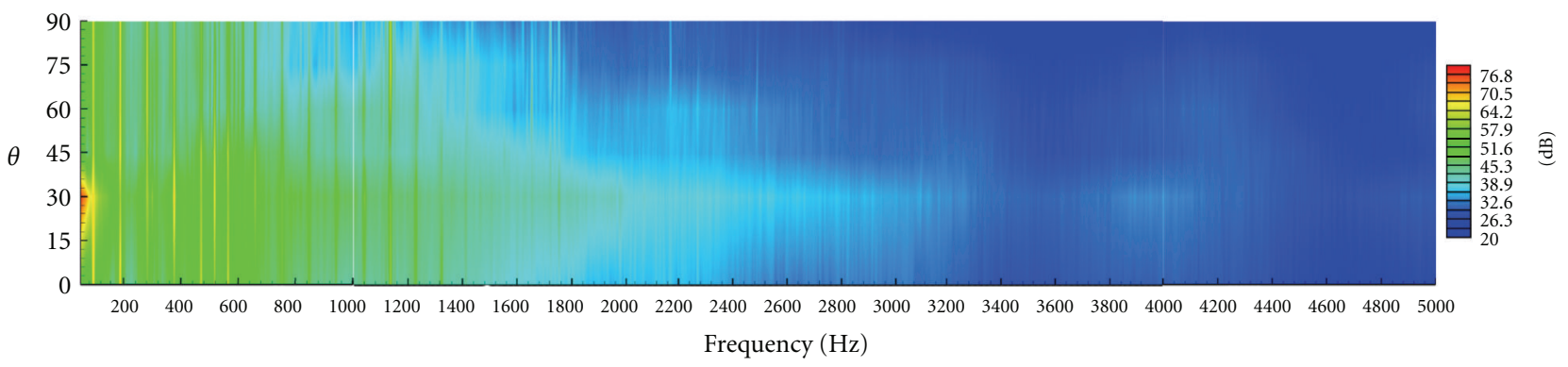

FIgURE 9: Directivity map of the $L_{p}$ far field autospectra for the MVB fan.

TABLE 2: Comparison of acoustic performance.

\begin{tabular}{lcccccc}
\hline \multirow{2}{*}{ Volume flow } & \multicolumn{2}{c}{ Datum fan } & \multicolumn{2}{c}{$T F$ fan } & \multicolumn{2}{c}{$M V B$ fan } \\
& Unweighted & A weighted & Unweighted & A weighted & Unweighted & A weighted \\
$\mathrm{m}^{2} / \mathrm{s}$ & $O L_{w}$ & $O L_{w}$ & $O L_{w}$ & $O L_{w}$ & $O L_{w}$ & $O L_{w}$ \\
\hline 8 & $\mathrm{~dB}$ & $\mathrm{~dB}(\mathrm{~A})$ & $\mathrm{dB}$ & $\mathrm{dB}(\mathrm{A})$ & $\mathrm{dB}$ & $\mathrm{dB}(\mathrm{A})$ \\
7 & 100,1 & 92,5 & 96,7 & 89,3 & 98,6 & 88,1 \\
6 & 96,8 & 92,5 & 96,6 & 87,9 & 94,7 & 86,7 \\
\hline
\end{tabular}

modification of the spectral behaviour immediately above the dominant tone at the BPF. Low-frequency tones similar to that which the datum produced appeared here only radiating to the pattern at $\theta=30$ degrees. When the frequencies reached the fifth BPF harmonic $(484.4 \mathrm{~Hz})$, the BPF harmonics radiated in the whole azimuthal sector. Other differences were noticeable in the range of frequencies from $600 \mathrm{~Hz}$ to $1.2 \mathrm{kHz}$, where the $M V B$ spectrum appeared to reduce the on-axis broadband $L_{p}$ level. Tonal spots emerged at high-order BPF harmonics, for example, seventh and eighth BPFs, radiating mostly off-axis ( $\theta=45-90$ degrees), because of the occurrence of a general background noise reduction in this sector [27]. Such a change would support the interpretation of this phenomenon as induced by the particular aerodynamic working of variable-thickness $M V B$ blade geometrics. Starting from $1.5 \mathrm{kHz}$, the map shows an abrupt reduction of the broadband noise as well as the tonal noise, being reduced from an average $L_{p}=50 \mathrm{~dB}$ down to $40 \mathrm{~dB}$, but two preferred radiation directions are visible about 30 degrees off-axis and laterally in the range $60-75$ degrees. Moving over the frequency $2 \mathrm{kHz}$, the tones observed above disappeared and the broadband noise was dominant. Nonetheless, the sector at $\theta=30$ degrees kept the noise's preferential radiation pattern up to the highest frequency range, showing constantly a sound pressure $L_{p}$ about $30 \mathrm{~dB}$. From this viewpoint it is possible to summarise that whilst the $T F$ featured the greatest tonal emission on the first BPF, the MVB had the least. This modifiedtip configuration broadband noise appeared similar to, but quieter than, the noise from the datum one in the low frequencies. Off the axis, at an angle of 90 degrees, the authors observed relatively comparable narrowband emissions for all three configurations. With regards to the broadband in this angular position, the TF and MVB spectra appeared to have $L_{p}$ levels which decreased more quickly when compared to the datum up to $5 \mathrm{kHz}$, whereas at higher frequency, the $M V B$ spectrum recovered the datum and the $T F$ sound pressure levels with a significant reduction of broadband noise emission.

\section{Conclusions}

The paper reports the results of an experimental study which assesses the acoustic improvement that occurs with three different fan impeller geometries. The authors also based the investigation on a developed technique to characterise noise sources along impeller's radius. The authors have realised the aim of the work, with the investigation characterising the noise sources spanwise along the tested fans' blades. The authors then established and discussed general acoustic proprieties of this family of fans.

The authors established tonal reduction, due to the enhanced blade-tip end-plates. They clarified the relevance of the tip features to influencing the radial distribution of the noise sources using coherence analysis. The modified multiple-vortex breakdown end-plate design was effective in reducing the broadband noise, improving the turbulent noise frequency range. The data that the authors obtained from the experiments demonstrated that the tip aeroacoustic emissions were differently sensitive when working in the presence of a single breakdown event, or multiple breakdowns as produced by the $M V B$.

The comparison of different tip features indicated that influence of the end-plate shaping positively impacted the fan blades' global noise emission to which they were applied. 


\section{Nomenclature}

\section{Latin Letters}

BPF: blade passing frequency $(\mathrm{Hz})$

$f: \quad$ frequency $(\mathrm{Hz})$

$K_{s}$ : $\quad$ specific noise level $(\mathrm{dB})$,

$K_{s}=O L_{w}-10 \log \left(V \cdot \Delta p_{\text {tot }}^{2}\right)$

l: $\quad$ blade chord

$L_{p}: \quad$ sound pressure level $(\mathrm{dB})$

$L_{w}$ : sound power level $(\mathrm{dB})$

$O L_{w}$ : overall sound power level (dB)

$\Delta p_{\text {tot }}$ : total pressure $(\mathrm{Pa})$

$t: \quad$ blade pitch

$V: \quad$ volume flow rate $\left(\mathrm{m}^{3} / \mathrm{s}\right)$.

\section{Greek Letters}

$\eta_{\text {tot }}$ : fan efficiency based on total pressure rise

$\chi$ : $\quad$ rotor tip clearance, $\%$ of the span.

\section{Acknowledgments}

The authors conducted the present research in the context of contract FW-DMA09-11 between Fläkt Woods Ltd and the Dipartimento di Ingegneria Meccanica e A. erospaziale, S. apienza University of Rome. Two of the authors (Bianchi and, Corsini) acknowledge the assistance of MIUR (the Italian Ministry for Academic Research) under the projects Ateneo and Facoltà 2009.

\section{References}

[1] S. E. Wright, "The acoustic spectrum of axial flow machines," Journal of Sound and Vibration, vol. 45, no. 2, pp. 165-223, 1976.

[2] N. A. Cumpsty, "Review: a critical review of turbomachinery noise," Journal of Fluids Engineering, vol. 99, no. 2, pp. 278293, 1977.

[3] T. Fukano, Y. Takamatsu, and Y. Kodama, "The effects of tip clearance on the noise of low pressure axial and mixed flow fans," Journal of Sound and Vibration, vol. 105, no. 2, pp. 291308, 1986.

[4] F. Holste and W. Neise, "Noise source identification in a propfan model by means of acoustical near field measurements," Journal of Sound and Vibration, vol. 203, no. 4, pp. 641-665, 1997.

[5] H. Marcinowski, "Einfluss des Laufradspalts und der Luftfuehrung bei einem Kuehlgeblaese axialer Bauart," Motortechnische Zeitschrift, vol. 14, pp. 259-262, 1953.

[6] B. D. Mugridge and C. L. Morfey, "Sources of noise in axial flow fan," Journal of the Acoustical Society of America, vol. 51, no. 5, pp. 1411-1426, 1972.

[7] R. E. Longhouse, "Control of tip-vortex noise of axial flow fans by rotating shrouds," Journal of Sound and Vibration, vol. 58, no. 2, pp. 201-214, 1978.

[8] T. Fukano and C. M. Jang, "Tip clearance noise of axial flow fans operating at design and off-design condition," Journal of Sound and Vibration, vol. 275, no. 3-5, pp. 1027-1050, 2004.
[9] F. Kameier and W. Neise, "Rotating blade flow instability as a source of noise in axial turbomachines," Journal of Sound and Vibration, vol. 203, no. 5, pp. 833-853, 1997.

[10] D. A. Quinlan and P. H. Bent, "High frequency noise generation in small axial flow fans," Journal of Sound and Vibration, vol. 218, no. 2, pp. 177-204, 1998.

[11] C. E. Jensen, “Axial-flow Fan," 1986, Patent No. US 4,630,993.

[12] C. M. L. Longet, "Axial Flow Fan with Noise Reducing Means," 2003, US Patent 2003/0123987 A1.

[13] M. Mimura, “Axial Flow Fan,” 2003, US Patent 6,648,598 B2.

[14] R. B. Uselton, L. J. Cook, and T. Wright, "Fan with Reduced Noise Generation,” 2005, US Patent 2005/0147496 A1.

[15] S. Bianchi, A. Corsini, F. Rispoli, and A. G. Sheard, "Detection of aerodynamic noise sources in low-speed axial fans with tip end-plates," Proceedings of the Institution of Mechanical Engineers C, vol. 223, no. 6, pp. 1379-1392, 2009.

[16] A. Corsini, F. Rispoli, and A. G. Sheard, "Development of improved blade tip end-plate concepts for low-noise operation in industrial fans," in Proceedings of the 13th International Conference on Modelling Fluid Flow Technologies, pp. 50-61, Budapest, Hungary, September 2006.

[17] A. Corsini, F. Rispoli, and A.G. Sheard, "A Meridional Fan," 2008, Patent No. GB 0800582.9.

[18] A. Corsini, F. Rispoli, and A. G. Sheard, "Development of improved blade tip endplate concepts for low-noise operation in industrial fans," Proceedings of the Institution of Mechanical Engineers A, vol. 221, no. 5, pp. 669-681, 2007.

[19] A. Corsini and A. G. Sheard, "Tip end-plate concept based on leakage vortex rotation number control," Journal of Computational and Applied Mechanics, vol. 8, no. 1, pp. 21-37, 2007.

[20] M. Inoue, M. Kuroumaru, and M. Fukuhara, "Behavior of tip leakage flow behind an axial compressor rotor," Journal of Engineering for Gas Turbines and Power, vol. 108, no. 1, pp. 714,1986

[21] A. Corsini and F. Rispoli, "Using sweep to extend the stall-free operational range in axial fan rotors," Proceedings of the Institution of Mechanical Engineers A, vol. 218, no. 3, pp. 129-139, 2004.

[22] S. Bianchi, A. Corsini, F. Rispoli, and A. G. Sheard, "Experimental aeroacoustic studies on improved tip geometries for passive noise signature control in low-speed axial fan," Transactions of the ASME, Journal of Vibration \& Acoustics, vol. 131, Article ID 061007, 2009.

[23] A. Corsini, F. Rispoli, and A. G. Sheard, "Shaping of tip endplate to control leakage vortex swirl in axial flow fans," Transactions of ASME, Journal of Turbomachinery, vol. 132, Article ID 031005, pp. 1-9, 2010.

[24] L. J. Leggat and T. E. Siddon, "Experimental study of the aeroacoustic mechanism of rotor-vortex interactions," Journal of the Acoustical Society of America, vol. 64, no. 4, pp. 1070-1077, 1978.

[25] B. Magliozzi, D. B. Hanson, B. V. Johnson, and F. B. Metzger, "Noise and wake structure measurements in a subsonic tip speed fan," Tech. Rep. CR-2323, NASA, 1973.

[26] M. R. Khourrami and M. Choudari, "A novel approach for reducing rotor tip-clearance induced noise in turbofan engines," in Proceedings of the 7th AIAA/CEAS AeroacousticsConference, Maastricht The Netherlands, May 2001.

[27] S. Bianchi, A. G. Sheard, A. Corsini, and F. Rispoli, "Far-field radiation of aerodynamic sound sources in axial fans fitted with passive noise control features," Transactions of the ASME, Journal of Vibration \& Acoustics, vol. 133, Article ID 051001, 2011. 
[28] P. D. Shah, D. Mobed, Z. Spakovszky, and T. F. Brooks, "Aero-acoustics of drag generating swirling exhaust flows," in Proceedings of the 13th AIAA/CEAS Aeroacoustics Conference (28th AIAA Aeroacoustics Conference), Rome, Italy, 2007, Paper No. AIAA-2007-3714. 

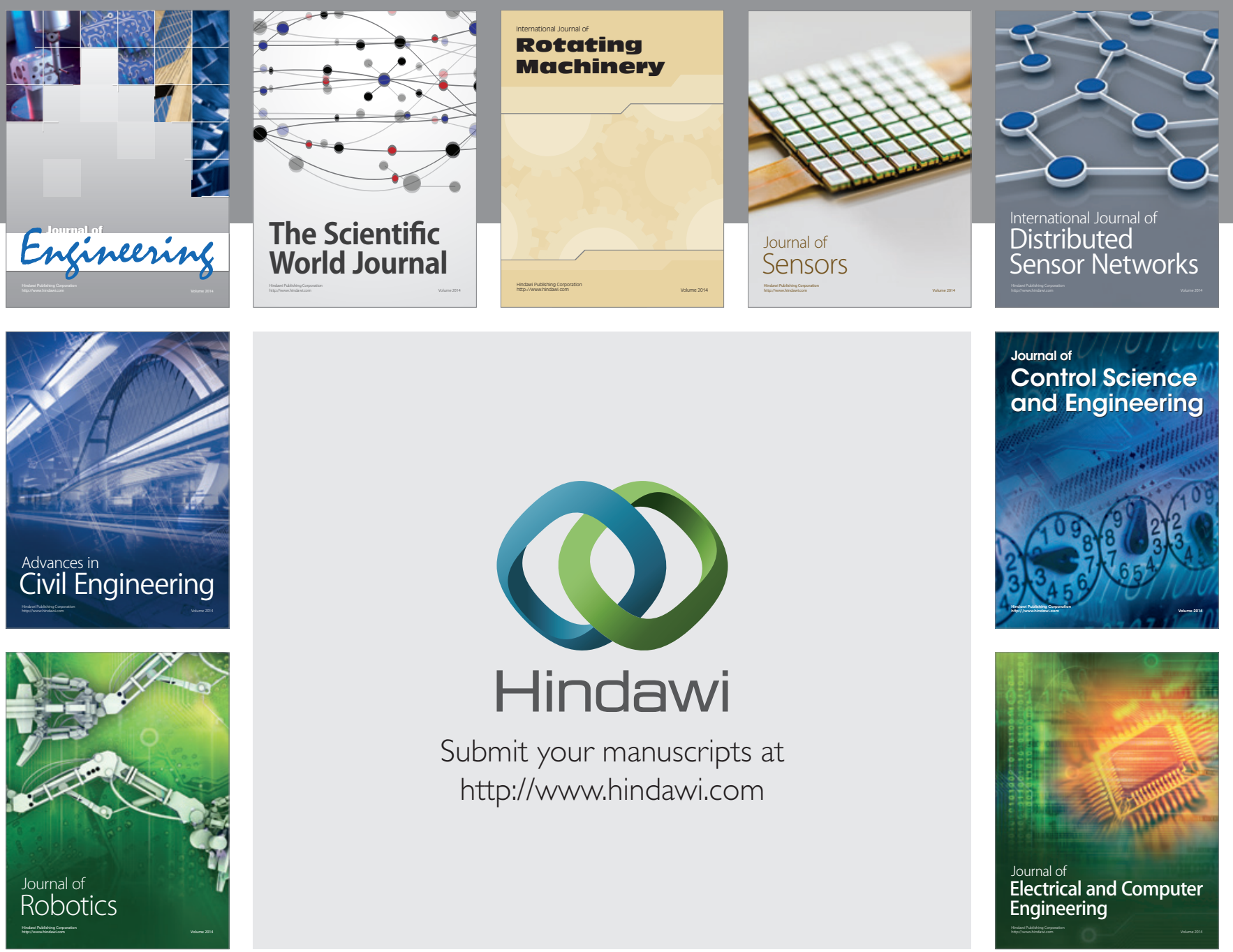

Submit your manuscripts at

http://www.hindawi.com
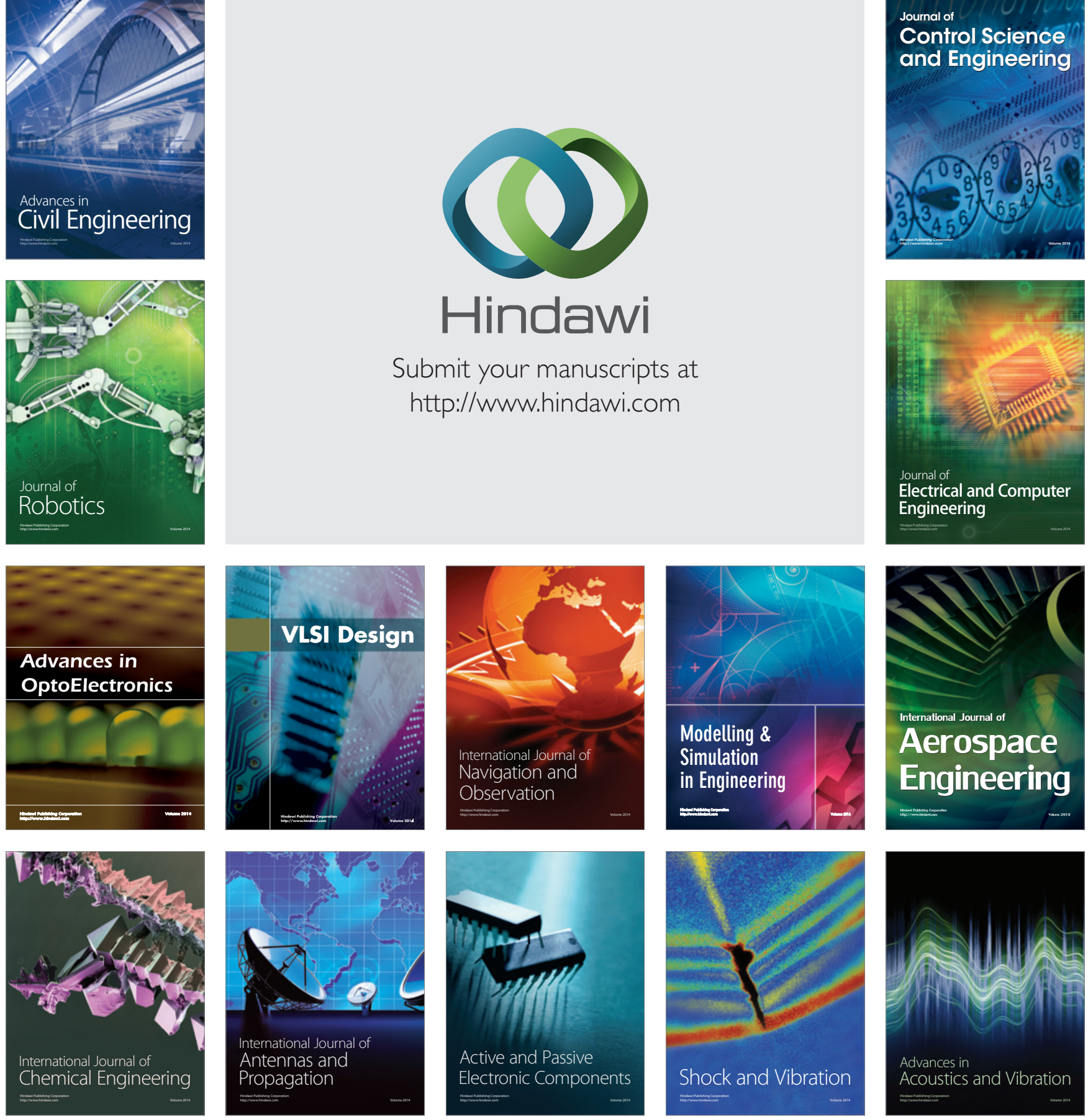